\title{
The Influence of Postoperative Tibiofemoral Alignment on the Clinical Results of Unicompartmental Knee Arthroplasty
}

\author{
Kyung Tae Kim, MD, Song Lee, MD, Tae Woo Kim, MD, Jung Soo Lee, MD and Kyung Hwan Boo, MD
}

Department of Orthopedic Surgery, Seoul Sacred Heart General Hospital, Seoul, Korea

\begin{abstract}
Purpose: To evaluate the influence of postoperative tibiofemoral alignment on the clinical results and failure in patients who underwent unicompartmental knee athroplasty (UKA).

Materials and Methods: We reviewed 246 cases of medial UKA which were followed up for at least 5 years after the operation. The clinical results were compared between 5 groups classified according to the tibiofemoral angle that was measured at 3 months after surgery. We analyzed the relationship between the tibiofemoral alignment and the failure after UKA.

Results: The preoperative tibiofemoral angle was changed from $0.4^{\circ}$ of varus to $5.4^{\circ}$ of valgus after surgery and the average correction angle was $5.8^{\circ}$. During the follow-up, which averaged 7 years and 5 months, the knee score and function score were improved significantly in all groups regardless of the tibiofemoral angle $(p<0.01)$. There were no significant difference between the groups in the clinical results $(p>0.05)$. However, there were significant differences in the cumulative survival rate of implants between the groups and the highest rate was found in the group with a tibiofemoral angle of $4^{\circ}$ to $6^{\circ}$ of valgus $(\mathrm{p}<0.01)$.

Conclusions: The tibiofemoral angle after UKA had no significant influence on the midterm clinical scores, but there was a significant relationship between the postoperative tibiofemoral angle and failure rate of implant.
\end{abstract}

Key words: Knee joint, Unicompartmental arthroplasty, Tibiofemoral angle.

\section{Introduction}

Among various factors that have been associated with clinical results and survivorship of unicompartmental knee arthroplasty (UKA), postoperative tibiofemoral alignment is crucial to the prevention of the progression of degenerative arthritis and implant-related complications. Thus, tibiofemoral axes have

Received April 25, 2011; Revised (1st) June 7, 2011;

(2nd) October 28, 2011; (3rd) December 21, 2011;

Accepted December 30, 2011.

Correspondence to: Tae Woo Kim, MD.

Department of Orthopedic Surgery, Seoul Sacred Heart General Hospital, 259 Wangsan-ro, Dongdaemun-gu, Seoul 130-011, Korea. Tel: +82-2-966-1616, Fax: +82-2-968-2394

Email:pasha96@naver.com

This is an Open Access article distributed under the terms of the Creative Commons Attribution Non-Commercial License (http://creativecommons.org/licenses/by-nc/3.0/) which permits unrestricted non-commercial use, distribution, and reproduction in any medium, provided the original work is properly cited. been used as a measure of the success of UKA and a predictor of revision $^{1,2)}$. Although neutral correction or slight undercorrection of the tibiofemoral angle is advocated in the literature to reduce UKA failure rates, the optimal level of correction still remains controversial. There is a paucity in the literature regarding the relationship between the postoperative tibiofemoral alignment and the clinical results and the survivorship of $\mathrm{UKA}^{3-6}$. In this study, we investigated the influence of the postoperative tibiofemoral alignment on the clinical results and survivorship of UKA by assessing clinical parameters including knee score, function score, and range of knee motion (ROM) and failure rates in patients classified according to the tibiofemoral angle measured three months postoperatively.

\section{Materials and Methods}

A total of 473 cases of medial UKA were performed between January 2002 and December 2004 at our institution. Of these, 246 cases (194 patients; male:female, 7:239) with a minimally invasive UKA using the Oxford phase 3 (Biomet Orthopedics Inc., Warsaw, IN, USA) prosthesis under the diagnosis of medial 
Table 1. Demographics

\begin{tabular}{|c|c|c|c|c|c|c|}
\hline Postoperative TFA & $\leq 0^{\circ}$ & $1^{\circ}-3^{\circ}$ & $4^{\circ}-6^{\circ}$ & $7^{\circ}-9^{\circ}$ & $\geq 10^{\circ}$ & Total \\
\hline Number of cases & 11 & 43 & 101 & 78 & 13 & 246 \\
\hline Number of failure & 1 & 5 & 2 & 5 & 4 & 17 \\
\hline Sex (male:female) & $0: 11$ & $1: 42$ & $3: 98$ & $3: 75$ & $0: 13$ & $7: 239$ \\
\hline Age (yr) & $58.1 \pm 5.2$ & $60.5 \pm 7.5$ & $61.6 \pm 7.4$ & $62.4 \pm 6.4$ & $62.5 \pm 9.0$ & $61.5 \pm 7.1$ \\
\hline Weight (kg) & $60 \pm 3.2$ & $61.6 \pm 7.1$ & $61.9 \pm 8.2$ & $62.6 \pm 6.8$ & $59.2 \pm 8.6$ & $61.8 \pm 7.4$ \\
\hline $\mathrm{F} / \mathrm{U}$ (month) & $97.6 \pm 7.2$ & $89.9 \pm 12.2$ & $88.7 \pm 15.9$ & $86.4 \pm 17.1$ & $94.0 \pm 17.2$ & $88.8 \pm 15.6$ \\
\hline
\end{tabular}

TFA: tibiofemoral angle, F/U: follow-up.

compartment degenerative arthritis were enrolled in this study. The minimum follow-up period was 5 years. The mean age of the patients was 62 years (range, 45 to 84 years) (Table 1).

The major indications for surgery were medial compartment degenerative arthritis without inflammation, normal function of the anterior/posterior cruciate ligament, $\leq 15^{\circ}$ varus deformity, $\leq 15^{\circ}$ flexion contracture, and $\geq 110^{\circ} \mathrm{ROM}$. An intraoperative finding of lateral compartment degenerative arthritis was the exclusion criterion, but we performed UKA regardless of the grade of degeneration of the patellofemoral joint unless symptoms including anterior knee pain or retropatellar area pain during stair climbing or squatting were present.

The patients were classified into 5 groups according to the tibiofemoral angle ( $\leq 0^{\circ}$ group, $1^{\circ}-3^{\circ}$ group, $4^{\circ}-6^{\circ}$ group, $7^{\circ}-9^{\circ}$ group, and $\geq 10^{\circ}$ group) measured on the anteroposterior (AP) weight-bearing radiograph obtained 3 months postoperatively as suggested by Kennedy and White $e^{5)}$ and Perkins and Gunckle ${ }^{2)}$. The design of this study was prospective. Preoperative and annual follow-up examinations were performed for clinical and radiographic assessment. The mean follow-up period was 7 years and 5 months (range, 5 years to 9 years and 1 month). For the clinical assessment, knee pain, ROM, knee score, and function score were assessed according to the Knee Society Clinical Rating System ${ }^{7}$. Radiographs were taken with the knee in full extension. For the radiographic assessment, bony change, implant loosening and wear, and dislocation were investigated. The tibiofemoral angle was defined as the acute angle between the longitudinal axis of the femoral shaft and that of the tibial shaft according to Bauer's method $^{8}$. Measurements were performed twice by one orthopedic surgeon and one researcher (nurse). The mean value of the two measurements was rounded off to a whole number (Fig. 1).

Statistical analysis was done using SPSS ver. 16.0 (SPSS Inc., Chicago, IL, USA). The preoperative and last follow-up knee score, function score, and ROM were compared using the Wilcoxon signed-rank test. Differences between the preoperative
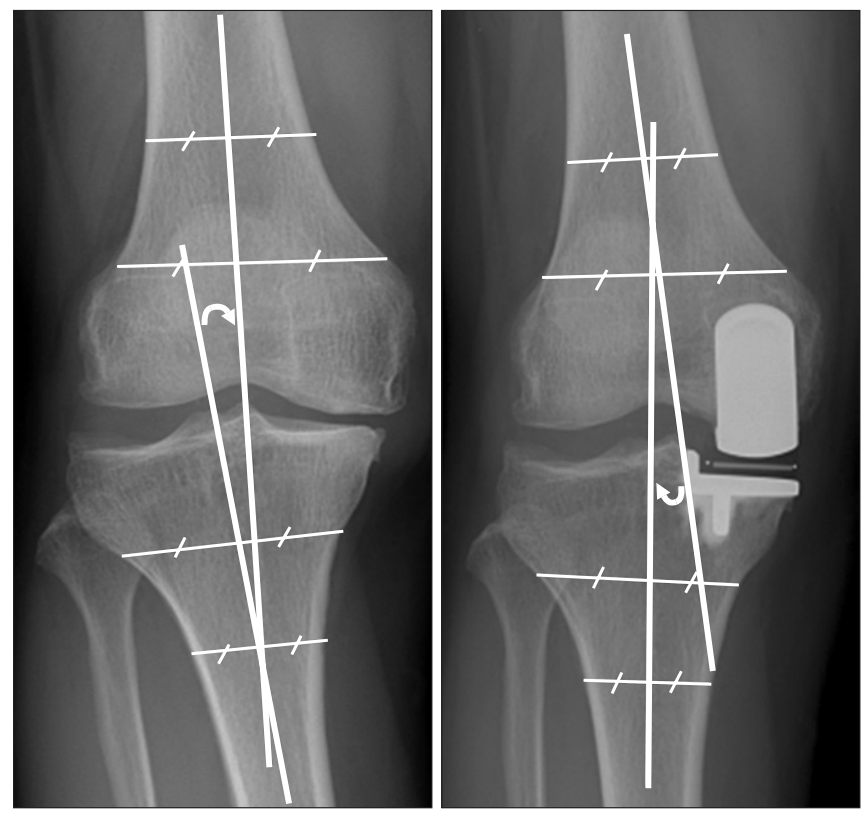

Fig. 1. Preoperative and postoperative anteroposterior radiographs after medial unicompartmental knee arthroplasty showing radiographic measurement method for tibiofemoral angle.

and postoperative knee score, function score, and ROM among groups classified according to the tibiofemoral angle were determined using the Kruskal-Wallis test. Change of the mobile bearing and revision operation were considered as a failure. The survival rate of the implants was calculated using the KaplanMeier method. The log-rank test was used to assess statistical differences among the groups. A $p<0.05$ was considered statistically significant.

\section{Results}

The mean tibiofemoral angle was changed from $0.4^{\circ}$ of varus (range, $8^{\circ}$ of varus to $10^{\circ}$ of valgus) preoperatively to $5.4^{\circ}$ of valgus (range, $2^{\circ}$ of varus to $12^{\circ}$ of valgus) postoperatively. The mean correction angle was $5.8^{\circ}$ (range, $0^{\circ}$ to $12^{\circ}$ ). The mean 
Table 2. Clinical Results

\begin{tabular}{|c|c|c|c|c|}
\hline & $\begin{array}{c}\text { Postoperative } \\
\text { TFA }\end{array}$ & Preoperative & Last F/U & p-value ${ }^{a)}$ \\
\hline \multirow{6}{*}{$\begin{array}{l}\text { Knee score } \\
\text { (KSS) }\end{array}$} & $\leq 0^{\circ}$ & $51.4 \pm 4.4$ & $73.4 \pm 7.6$ & 0.003 \\
\hline & $1^{0}-3^{\circ}$ & $50.3 \pm 9.9$ & $80.9 \pm 7.0$ & 0.000 \\
\hline & $4^{0}-6^{\circ}$ & $53.1 \pm 9.6$ & $87.7 \pm 8.5$ & 0.000 \\
\hline & $7^{\circ}-9^{\circ}$ & $55.0 \pm 9.5$ & $88.3 \pm 7.6$ & 0.000 \\
\hline & $\geq 10^{\circ}$ & $59.1 \pm 8.2$ & $84.1 \pm 14.1$ & 0.002 \\
\hline & p-value $e^{b)}$ & 0.007 & 0.000 & - \\
\hline \multirow{6}{*}{$\begin{array}{l}\text { Corrected knee } \\
\text { score }\end{array}$} & $\leq 0^{\circ}$ & $71.4 \pm 4.4$ & $90.6 \pm 7.4$ & 0.003 \\
\hline & $1^{\mathrm{o}}-3^{\mathrm{o}}$ & $68.3 \pm 14.3$ & $90.0 \pm 6.6$ & 0.000 \\
\hline & $4^{0}-6^{\circ}$ & $68.8 \pm 8.3$ & $88.5 \pm 8.4$ & 0.000 \\
\hline & $7^{\circ}-9^{\circ}$ & $68.3 \pm 9.6$ & $88.3 \pm 7.6$ & 0.000 \\
\hline & $\geq 10^{\circ}$ & $66.0 \pm 10.1$ & $85.8 \pm 14.2$ & 0.003 \\
\hline & p-value $e^{\mathrm{b})}$ & 0.487 & 0.477 & - \\
\hline \multirow{6}{*}{$\begin{array}{l}\text { Function score } \\
\text { (KSS) }\end{array}$} & $\leq 0^{\circ}$ & $62.7 \pm 9.0$ & $79.1 \pm 10.4$ & 0.010 \\
\hline & $1^{\mathrm{o}}-3^{\mathrm{o}}$ & $54.7 \pm 10.5$ & $82.6 \pm 10.0$ & 0.000 \\
\hline & $4^{\circ}-6^{\circ}$ & $55.6 \pm 10.6$ & $82.2 \pm 12.5$ & 0.000 \\
\hline & $7^{\circ}-9^{\circ}$ & $55.7 \pm 11.8$ & $80.3 \pm 12.3$ & 0.000 \\
\hline & $\geq 10^{\circ}$ & $54.2 \pm 12.6$ & $81.2 \pm 17.1$ & 0.000 \\
\hline & $\mathrm{p}$-value $\mathrm{e}^{\mathrm{b})}$ & 0.161 & 0.636 & - \\
\hline \multirow{6}{*}{$\begin{array}{l}\text { Range of knee } \\
\text { motion }\end{array}$} & $\leq 0^{\circ}$ & $133.2 \pm 2.5^{\circ}$ & $133.6 \pm 4.5^{\circ}$ & 0.705 \\
\hline & $1^{\mathrm{o}}-3^{\mathrm{o}}$ & $129.4 \pm 10.4^{\circ}$ & $133.3 \pm 5.8^{\circ}$ & 0.000 \\
\hline & $4^{0}-6^{\circ}$ & $126.2 \pm 12.3^{\circ}$ & $131.8 \pm 9.1^{\circ}$ & 0.000 \\
\hline & $7^{\circ}-9^{\circ}$ & $127.1 \pm 15.9^{\circ}$ & $133.3 \pm 4.5^{\circ}$ & 0.000 \\
\hline & $\geq 10^{\circ}$ & $124.2 \pm 14.3^{\circ}$ & $128.8 \pm 11.9^{\circ}$ & 0.172 \\
\hline & p-value $e^{\text {b) }}$ & 0.085 & 0.148 & - \\
\hline
\end{tabular}

Values are presented as mean \pm standard deviation. TFA: tibiofemoral angle, KSS: Knee Society score, F/U: follow-up.

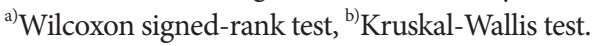

knee score and function score were improved from 53.4 points (range, 20 points to 70 points) and 55.7 points (range, 25 points to 80 points) preoperatively to 85.9 points (range, 50 points to 100 points) and 81.5 points (range, 60 points to 100 points) at the last follow-up. The mean ROM increased from $127.2^{\circ}$ (range, $85^{\circ}$ to $135^{\circ}$ ) preoperatively to $132.6^{\circ}$ (range, $85^{\circ}$ to $135^{\circ}$ ) at the last follow-up $(\mathrm{p}<0.001)$. The knee score and function score were improved significantly at the last follow-up regardless of the tibiofemoral angle in all groups $(\mathrm{p}<0.01)$. The postoperative tibiofemoral angle was found to be related to the pre- and postoperative knee score. However, when the deductions for tibiofemoral angle made according to the Knee Society Clinical Rating System were adjusted for comparison purposes, no significant relationship could be found between the tibiofemoral angle and the knee score, function score, and ROM ( $>0.05)$ (Table 2).

There were 17 failures including isolated dislocation of the mobile bearing $(n=8)$, medial collateral ligament injury combined with dislocation of the mobile bearing $(\mathrm{n}=1)$, femoral component loosening $(n=1)$, tibial component loosening $(n=1)$, femoral component loosening combined with dislocation of the mobile bearing $(n=3)$, tibial plateau fracture $(n=1)$, deep infection $(n=1)$, and wearing with fracture of the mobile bearing $(n=1)$. So, the cumulative survival rate of the implants was $92.3 \%$ at 8 years $(95 \%$ confidence interval [CI], 88.8-95.8\%). The number of failures in each group classified according to the postoperative tibiofemoral angle was 1 in the $\leq 0^{\circ}$ group ( $\left.n=11\right), 5$ in the $1^{\circ}-3^{\circ}$ group ( $n=43$ ), 2 in the $4^{\circ}-6^{\circ}$ group $(\mathrm{n}=101), 5$ in the $7^{\circ}-9^{\circ}$ group $(\mathrm{n}=78)$, and 4 in the $\geq 10^{\circ}$ group ( $n=13$ ). The cumulative survival rate for each group was the highest in the $4^{\circ}-6^{\circ}$ of valgus group and the lowest in the $\geq 10^{\circ}$ of valgus group (log-rank test, $\mathrm{p}<0.01$ ) (Table 3, Fig. 2).

Table 3. Survivorship of Implant according to Postoperative Tibiofemoral Angle (TFA)

\begin{tabular}{|c|c|c|c|c|c|c|}
\hline Postoperative TFA & Total number & Number of events & $\begin{array}{c}\text { Number of } \\
\text { censored }\end{array}$ & $\begin{array}{c}\text { Percent of } \\
\text { censored (\%) }\end{array}$ & 8 yr-survival rate (\%) & $\begin{array}{c}95 \% \text { confidence } \\
\text { interval (\%) }\end{array}$ \\
\hline$\leq 0^{\circ}$ & 11 & 1 & 10 & 90.9 & 90.9 & $73.8-100$ \\
\hline $1^{\mathrm{o}}-3^{\mathrm{o}}$ & 43 & 5 & 38 & 88.4 & 87.0 & $76.2-97.8$ \\
\hline $4^{\circ}-6^{\circ}$ & 101 & 2 & 99 & 98.0 & 97.8 & $94.9-100$ \\
\hline $7^{\circ}-9^{\circ}$ & 78 & 5 & 73 & 93.6 & 92.9 & $86.8-99.0$ \\
\hline$\geq 10^{\circ}$ & 13 & 4 & 9 & 69.2 & 69.2 & $44.1-94.3$ \\
\hline Total & 246 & 17 & 229 & 93.1 & 92.3 & $88.8-95.8$ \\
\hline p-value ${ }^{a)}$ & & & & & 0.001 & \\
\hline
\end{tabular}

${ }^{a)}$ Log-rank test. 


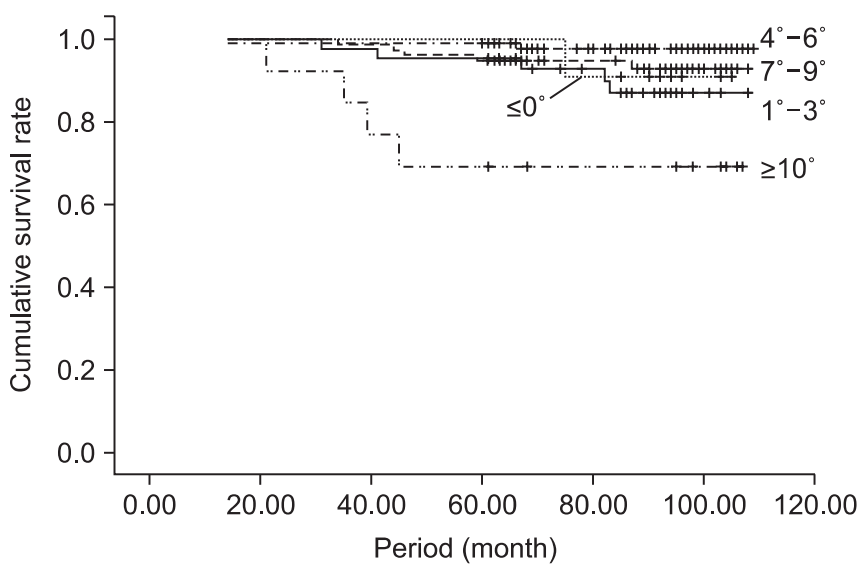

Fig. 2. Kaplan-Meier survivorship analysis curve shows higher cumulative survival rate of unicompartmental knee arthroplasty in knees with a tibiofemoral angle of $4^{\circ}-6^{\circ}$ of valgus.

\section{Discussion}

UKA is a surgical procedure to replace one of the three compartments for treatment of osteoarthritis of the knee. First introduced in the 1950s, UKA has been widely used since the 1970s. In the absence of proper prosthesis design, surgical equipment and patient selection criteria, UKA resulted in high failure rates and fell out of favor until the mid-1990s. Thereafter, however, renewed interest in UKA has been generated by favorable results with the introduction of minimally invasive surgical techniques, establishment of indications, and continuous improvement in prostheses and in surgical equipment ${ }^{9-13)}$.

Proper patient selection, proven implant designs and accurate surgical techniques are critical to the success of UKA. Factors that may influence the clinical results and survivorship of UKA include age and weight of patients, type and position of implants, composition and thickness of the polyethylene component, and tibiofemoral alignment ${ }^{11,14-16)}$. In particular, postoperative tibiofemoral alignment is an important factor in total knee arthroplasty (TKA) and various tibiofemoral axes have been considered as a measure of the success of TKA and a predictor of revision ${ }^{5,15,17)}$. The normal tibiofemoral angle has been reported as $5^{\circ}-8^{\circ}$ of valgus and accordingly, $7^{\circ}$ of valgus has been considered as the ultimate goal in TKA ${ }^{17-19)}$. For UKA, neutral correction or slight undercorrection has been recommended because overcorrection may increase the risk of degeneration in the opposite compartment whereas undercorrection may accelerate polyethylene wear and recurrence of deformity ${ }^{3-6}$.

In this study, we classified the patients according to the tibiofemoral angle measured on the AP weight-bearing radiographs taken at 3 months after UKA into 5 groups, $\leq 0^{\circ}$ group, $1^{\circ}-3^{\circ}$ group, $4^{\circ}-6^{\circ}$ group, $7^{\circ}-9^{\circ}$ group, and $\geq 10^{\circ}$ group. On the clinical assessment, the knee score and function score were improved in all groups during the mean 7 years and 5 months of midterm follow-up. No significant intergroup differences were found in the knee score, function score, and ROM. A correlation was found between the postoperative tibiofemoral angle and implant failure: the cumulative survival rate was the highest in the group with a postoperative tibiofemoral angle of $4^{\circ}-6^{\circ}$ of valgus and the lowest in the group with $\geq 10^{\circ}$ of valgus. Regarding the relationship between the tibiofemoral alignment after UKA and the clinical results (knee score and function score) and the survival rate, Kennedy and White ${ }^{5)}$ reported that superior clinical results were obtained when the mechanical axis fell in the center of the knee or slightly medial to the center. In the minimum 4-year follow-up study of 47 cases of fixed-bearing UKA by Jung et al. ${ }^{20}$, there were no differences in the clinical outcome between the more than $2^{\circ}$ varus corrected group and the under $2^{\circ}$ varus corrected group, but three failure cases were observed in the undercorrected group only. According to the study by Perkins and Gunckle ${ }^{2}$, clinical results were poor and revision rate was high when the postoperative tibiofemoral angle at 6 years after UKA was more than $3^{\circ}$ varus or more than $7^{\circ}$ valgus. Cahue et al. ${ }^{21)}$ reported that varus-valgus alignment of the knee was associated with the progression of patellofemoral osteoarthritis.

Knee arthroplasty has been known to provide good results through resurfacing of the involved compartment and correction of varus deformity. However, UKA may not be conducive to tibiofemoral angle correction because medial release should be minimized for ligament stability and soft tissue tension should be equal in flexion and extension. In addition, minimally invasive UKA may not be as accurate as open $\mathrm{UKA}^{3,22}$. In UKA, tibiofemoral alignment is determined by the height of the contact point between the medial femoral condyle and the tibial component, which is dependent on the implant design, resection level of the proximal tibia, ligament stability, preoperative deformity, implant thickness, and surgical technique ${ }^{3,4,19)}$. Therefore, meticulous care should be taken to proper implant selection based on the patient's preoperative condition and planning and execution of surgery in order to achieve the desired postoperative tibiofemoral angle as much as possible $e^{5)}$. Furthermore, continuous long-term follow-up should be carried out to look for possible varus deformity that may be caused by polyethylene wear even in knees with proper immediate postoperative tibiofemoral alignment $t^{4}$.

One of the limitations of this study is that the deductions 
applied to the tibiofemoral angle according to the Knee Society Clinical Rating System may have adversely influenced the intergroup comparison results. In an attempt to improve the accuracy of the results, we had to make some adjustments to the deductions. In addition, we think that some authors might question the use of anatomical axis, not the mechanical axis, for group classification in this study. Finally, the mean 7 years and 5 months of follow-up period was short to draw a definite conclusion. Therefore, further long-term follow-up is warranted.

\section{Conclusions}

We analyzed the clinical results and survival rate of medial UKA in patients with degenerative arthritis of the knee. During the mean follow-up of 7 years and 5 months, no relationship was found between the postoperative tibiofemoral alignment and the midterm clinical results including the knee score, function score, and ROM. However, the alignment was related to the implant survival rate: the cumulative survival rate was the highest in the $4^{\circ}-6^{\circ}$ of valgus group and the lowest in the $\geq 10^{\circ}$ of valgus group. Therefore, our results suggest that a postoperative tibiofemoral angle of $4^{\circ}-6^{\circ}$ of valgus is desirable and overcorrection should be avoided.

\section{References}

1. Cossey AJ, Spriggins AJ. The use of computer-assisted surgical navigation to prevent malalignment in unicompartmental knee arthroplasty. J Arthroplasty. 2005;20:29-34.

2. Perkins TR, Gunckle W. Unicompartmental knee arthroplasty: 3- to 10-year results in a community hospital setting. J Arthroplasty. 2002;17:293-7.

3. Fisher DA, Watts M, Davis KE. Implant position in knee surgery: a comparison of minimally invasive, open unicompartmental, and total knee arthroplasty. J Arthroplasty. 2003;18(7 Suppl 1):2-8.

4. Hernigou P, Deschamps G. Alignment influences wear in the knee after medial unicompartmental arthroplasty. Clin Orthop Relat Res. 2004;(423):161-5.

5. Kennedy WR, White RP. Unicompartmental arthroplasty of the knee. Postoperative alignment and its influence on overall results. Clin Orthop Relat Res. 1987;(221):278-85.

6. Ridgeway SR, McAuley JP, Ammeen DJ, Engh GA. The effect of alignment of the knee on the outcome of unicompartmental knee replacement. J Bone Joint Surg Br. 2002;84: 351-5.
7. Insall JN, Dorr LD, Scott RD, Scott WN. Rationale of the Knee Society clinical rating system. Clin Orthop Relat Res. 1989;(248):13-4.

8. Bauer GC, Insall J, Koshino T. Tibial osteotomy in gonarthrosis (osteo-arthritis of the knee). J Bone Joint Surg Am. 1969;51:1545-63.

9. Berger RA, Della Valle CJ. Unicompartmental knee arthroplasty: indications, techniques, and results. Instr Course Lect. 2010;59:47-56.

10. Kim KT, Lee S, Park HS, Cho KH, Kim KS. A prospective analysis of Oxford phase 3 unicompartmental knee arthroplasty. Orthopedics. 2007;30(Suppl 5):15-8.

11. Price AJ, Waite JC, Svard U. Long-term clinical results of the medial Oxford unicompartmental knee arthroplasty. Clin Orthop Relat Res. 2005;(435):171-80.

12. Repicci JA, Hartman JF. Minimally invasive unicondylar knee arthroplasty for the treatment of unicompartmental osteoarthritis: an outpatient arthritic bypass procedure. Orthop Clin North Am. 2004;35:201-16.

13. Saccomanni B. Unicompartmental knee arthroplasty: a review of literature. Clin Rheumatol. 2010;29:339-46.

14. Collier MB, Eickmann TH, Sukezaki F, McAuley JP, Engh GA. Patient, implant, and alignment factors associated with revision of medial compartment unicondylar arthroplasty. J Arthroplasty. 2006;21(6 Suppl 2):108-15.

15. Emerson RH Jr, Higgins LL. Unicompartmental knee arthroplasty with the oxford prosthesis in patients with medial compartment arthritis. J Bone Joint Surg Am. 2008; 90:118-22.

16. Kim KT, Lee S, Bae EH, Choi DJ, Park HS, Cho KH. Factors affecting the tibiofemoral alignment in minimally invasive unicompartmental knee arthroplasty. J Korean Orthop Assoc. 2006;41:148-55.

17. Chauhan SK, Scott RG, Breidahl W, Beaver RJ. Computerassisted knee arthroplasty versus a conventional jig-based technique. A randomised, prospective trial. J Bone Joint Surg Br. 2004;86:372-7.

18. Coventry MB. Osteotomy about the knee for degenerative and rheumatoid arthritis. J Bone Joint Surg Am. 1973;55:2348.

19. Iesaka K, Tsumura H, Sonoda H, Sawatari T, Takasita M, Torisu $\mathrm{T}$. The effects of tibial component inclination on bone stress after unicompartmental knee arthroplasty. J Biomech. 2002;35:969-74.

20. Jung WB, Song EK, Seon JK, Park SJ, Seo CY. Short-term outcomes of Miller Galante ${ }^{\circledR}$ fixed-bearing unicondylar knee 
90 Kim et al. The Influence of Postoperative Tibiofemoral Alignment on UKA

arthroplasty: a comparative study in outcomes according to postoperative correction angle. J Korean Orthop Assoc. 2010;45:426-32.

21. Cahue S, Dunlop D, Hayes K, Song J, Torres L, Sharma L. Varus-valgus alignment in the progression of patellofemoral osteoarthritis. Arthritis Rheum. 2004;50:2184-90.

22. Saito T, Takeuchi R, Yamamoto K, Yoshida T, Koshino T. Unicompartmental knee arthroplasty for osteoarthritis of the knee: remaining postoperative flexion contracture affecting overall results. J Arthroplasty. 2003;18:612-8. 